\title{
Understanding the water uptake in F-161 glass-epoxy composites using the techniques of luminescence spectroscopy and FT-NIR
}

\author{
Rita Sales ${ }^{1,2 *}$, Gilmar Thim² and Deborah Brunelli² \\ ${ }^{1}$ Faculdade de Tecnologia - FATEC, São José dos Campos, SP, Brazil \\ ${ }^{2}$ Chemistry Department, Instituto Tecnológico de Aeronáutica - ITA, São José dos Campos, SP, Brazil \\ *rita.sales@fatec.sp.gov.br
}

\begin{abstract}
This paper investigates the application of the luminescence spectroscopy technique in steady-state to study the moisture influence in glass fiber/epoxy prepreg and their laminates. The studies were monitored by intrinsic luminescence comparing the results with gravimetric analysis and near infrared with Fourier transform. Samples are cured and submitted to humidity controlled at 60 and $80^{\circ} \mathrm{C}$ until 90 days. It is verified that the decrease in the maximum emission of the samples is directly related to the material moisture content. However, for very short periods, there is an increase in the relative intensity and blue shift of the emission band for all samples treated at $60{ }^{\circ} \mathrm{C}$, which is related to an increase of the rigidity of the polymeric matrix. The results in this paper have a great significance because it brings a wide discussion on the interaction of water in epoxy composites materials.
\end{abstract}

Keywords: F-161 laminates, FT-NIR, luminescence spectroscopy, water uptake.

\section{Introduction}

The composite materials present in the aircraft structures are exposed to environmental conditions with elevated levels of moisture. The composite materials can absorb water molecules and this chemical interaction can result in a delamination process ${ }^{[1]}$. The delamination process consists on separating the layers resulted from interlaminar tensions.

The study of the water interaction with the polymer matrix at the molecular level is very important since the water uptake will determine the mechanical strength of the material at the macroscopic level. These materials are used in primary structural parts of aircraft under great mechanical stress. The knowledge of the mechanisms which drive moisture sorption, as well as of the influence of sample dimensions, temperature and relative humidity, becomes crucial when the long-term properties of the material are needed ${ }^{[2]}$.

Many researches have been done to understand the behavior of water in epoxy resin and composites, since this cured polymeric matrix contains strongly polar groups, such as hydroxyl groups and amine groups ${ }^{[3-6]}$. Adamson ${ }^{[6]}$ has done experiments of thermal expansion of two graphite/epoxy composite systems and concluded that water is initially absorbed and diffuses into the resin; then it begins to occupy polymer free volume, causing no swelling, while some of the water disrupts intermolecular hydrogen bonds, causing swelling by hydrogen bonding with the resin. In the final stage of water absorption, the swelling efficiency drops far below that observed in the initial stage. Apicella et al. ${ }^{[2]}$ have analyzed epoxy resin cured with amine compounds using DSC. These authors proposed three sorption mechanisms: (1) bulk dissolution of the water in the polymer network; (2) adsorption on hydrophilic sites and (3) adsorption on the surfaces of free volume elements.
Li et al. ${ }^{[7]}$ used time-resolved attenuated total reflection Fourier transform infrared spectroscopy (ATR-IR), near-infrared (NIR) spectroscopy and positron annihilation lifetime spectroscopy (PALS) to investigate water diffusion processes and the state of water molecules in six different epoxy resins. These authors concluded that water diffusion is controlled by local chain reorientation and bond dissociation of water molecules from epoxy networks. Four types of water molecules were detected: nonbonded, single bonded and two types of double hydrogen bonded.

Cotugno et al. ${ }^{[8]}$ e Roy et al. ${ }^{[9]}$ showed that water molecules can affect the epoxy matrices by (a) plasticization, (b) changes in physical properties and (c) hygrothermal degradation. The plasticization effects have been previously discussed. The changes in physical properties induce a decrease of yield strength and change of yield/deformation mechanisms. The hygrothermal degradation is characterized by micro-cracking, aging, breakdown of the polymer chain and the degradation of the fiber-matrix interface in polymer composites.

Usually, water absorption by the epoxy resin decreases the glass transition temperature $(\mathrm{Tg})$ due to the plasticization of the polymer network. This effect can lead to a decrease of the mechanical performance of the composite material. Those properties related to the compressive strength and intralaminar/interlaminar shear is significantly affected by the moisture absorbed, especially at high temperatures ${ }^{[10-12]}$. According to $\mathrm{Asp}^{[13]}$, the water absorption can cause interlaminar delamination or damage in carbon-epoxy composites.

One of the most important hygrothermal aging processes of the epoxy composites involves molecular interactions between water and epoxy resin, such as the plasticization 
phenomenon of the molecular network. This process may be reversible, but can lead to irreversible loss of mechanical properties ${ }^{[14]}$. According to Majerus et al. ${ }^{[15]}$, this phenomenon is related to the presence of water molecules in the matrix microcavities, by the interaction between water molecules with specific segments of the polymeric matrix. Epoxy resins can absorb water through three different ways: (a) bulk dissolution of water in polymer network, (b) adsorption on the hydrophilic sites and (c) adsorption in the voids. The transport of moisture below the Tg consists of a three-step process. First, the water occupies the voids of the polymer matrix. Next, the water molecules bind to the polymer network by hydrogen bonds, causing swelling. Finally, water diffuses into the densely crosslinked resin. Polarity and topology of the structure of crosslinked epoxy resin influence the adsorption of water ${ }^{[16]}$.

The effects of water absorption on the epoxy resin have been widely investigated by several techniques. However, none of them is sensitive and non-destructive for monitoring the moisture aging effects on composite materials like luminescence spectroscopy ${ }^{[17-21]}$. Sung and Sung ${ }^{[17,21]}$ used luminescence spectroscopy, intrinsic and extrinsic methods, to characterize the water uptake and the curing extension in composite materials. The intrinsic method involved the study of the emission of the resin matrix itself, while the extrinsic one consisted in adding a luminescent probe in the resin. These researchers noticed that there was some spectral shift in the wavenumber and/or some decrease in intensity of the emission spectra when water molecules were uptaken.

In this work, it was used glass fiber/epoxy prepreg F161 $\left(\right.$ Hexcel $^{\circledR}$ ), a composite system that is widely used in aeronautics industry in the manufacture of structural parts. The moisture effect on glass fiber/epoxy prepreg and glass fiber/epoxy laminates was studied by luminescence spectroscopy in steady-state condition using the intrinsic fluorescence method. The results were compared to Fourier Transform Near Infrared Spectroscopy (FT-NIR) and the sample weighting was performed according to ASTM D 5229/D 5229M-12 ${ }^{[22]}$. The changes in the maximum emission of the samples is directly related to the moisture content of the material. Other authors had not previously observed this photophysical behavior.

\section{Materials and Methods}

\subsection{Sample preparation}

These experiments used a plain wave glass fiber/epoxy resin prepreg, F161 (Hexcel Composites Ltd., Livermor, $\mathrm{CA}$ ), with size of $30 \mathrm{~mm} \times 20 \mathrm{~mm}$. Some prepreg samples were analyzed as received to understand their photophysical behavior and they were named as btt-pre (btt= before thermal treatment and "pre"= prepreg). The curing process was made according to the manufacturer's instructions. The prepreg samples were heated from room temperature to $177^{\circ} \mathrm{C}$ at a heating rate of $2^{\circ} \mathrm{C} / \mathrm{min}$, and then, they were maintained in this temperature for $120 \mathrm{~min}$. After this time, the samples were removed from the oven and maintained at room temperature for $20 \mathrm{~min}$. Samples submitted to this thermal treatment were named as $\mathrm{tt}$-pre ( $\mathrm{tt}=$ thermal treatment).
A set of desiccators was maintained at 60 and at $80^{\circ} \mathrm{C}$. Beckers with saturated aqueous solution of $\mathrm{NaBr}, \mathrm{NaCl}$ and $\mathrm{KCl}$ (Merck) were added to these desiccators to simulate a relative humidity of 58, 75 and $84 \%$, respectively. Pieces of samples already thermally treated were put onto glass plates next to the salt solution inside of each desiccator. The samples were maintained for 1, 7, 15,30, 60 and 90 days in each desiccator. The samples obtained after exposure to different environmental conditions were named pre-relative humidity-T-t, where "pre", relative humidity, T and t are, respectively, prepreg, relative humidity value, temperature and time, for example, pre-75-60-30 stands for a cured F-161 prepreg sample exposed to a relative humidity of $75 \%$ at $60{ }^{\circ} \mathrm{C}$ for 30 days.

To manufacturing the laminate samples, the prepreg layers were laid down in a plain mold forming a rectangular panel $(160 \mathrm{~mm} \times 160 \mathrm{~mm})$ with 14 layers. The curing process was running using a hydraulic press (SOLAB) at a pressure of $50 \mathrm{kgf} / \mathrm{cm}^{2}$ and $177^{\circ} \mathrm{C}$ using a heating rate of $2^{\circ} \mathrm{C} / \mathrm{min}$ and then maintained at this temperature for $120 \mathrm{~min}$. After that, samples were cut in pieces of $25 \mathrm{~mm} \times 15 \mathrm{~mm}$ and added to desiccators for $1,7,15$ and 30 days. The laminated samples were exposed to the same environmental conditions as the prepreg samples into desiccators. The samples obtained after the exposure to different environmental conditions were named lam-relative humidity-T-t, where lam stands for a cured laminate sample constituted of 14 prepregs. The remaining labels are similar to pre- relative humidity -T- $t$ that was previously described.

\subsection{Water absorption analysis}

The water absorption $\left(\% \mathrm{H}_{2} \mathrm{O}_{\text {abs }}\right)$ was determined using Equation 1, according to ASTM D 5229/D 5229M-12 ${ }^{[22]}$ :

$$
\% \mathrm{H}_{2} \mathrm{O}=\frac{\left(\mathrm{M}_{\mathrm{f}}-\mathrm{M}_{\mathrm{i}}\right)}{\mathrm{M}_{\mathrm{i}}} \mathrm{x} 100
$$

where $M_{F}$ and $M_{I}$ are, respectively, the mass of the samples after and before being added into the desiccators.

\subsection{Water diffusion}

The diffusion coefficients are easily calculated from the moisture-uptake profiles with Fickian diffusion ${ }^{[23]}$ (Equation 2):

$$
\frac{M_{t}}{M_{\infty}}=4\left(\frac{D t}{h^{2}}\right)^{1 / 2}\left[\frac{1}{\pi^{1 / 2}+2} \sum_{n=1}^{\infty}(-1)^{n} \operatorname{ierfc} \frac{n h}{2(D t)^{1 / 2}}\right]
$$

where $M_{T}$ is the moisture uptake at time $t, M_{\infty}$ is the equilibrium moisture uptake, $h$ is the sample thickness, $D$ is the diffusion coefficient, ierfc is the integral error function of each element and $\mathrm{n}$ is the concentration starting at 0 at the surface and spreading infinitely deep in the material. This solution of Fick's second law holds true for the conditions of an infinite sheet with constant penetrant activity on both sides of the sheet and a concentration independent of $D$. At very short times, where $M_{T} / M_{\infty}$ is less than 0.5 , Equation 2 can be defined approximated by the following (Equation 3):

$$
\frac{M_{t}}{M_{\infty}}=\frac{4}{h} \sqrt{\frac{D t}{\pi}}
$$


Equation 3 is easily rearranged to reveal that the initial slope in a plot of $M t / M$ versus $=t / h$ is related to the diffusion coefficient $D$ through the following (Equation 4):

$$
D=\frac{\pi}{16}\left(\frac{M_{t} / M_{\infty}}{\frac{\sqrt{t}}{h}}\right)^{2}
$$

\subsection{Infrared spectroscopy in the near region}

The infrared spectroscopy was also used to analyze the water content in the samples. The samples were analyzed in the near region using a Spectrum One Perkin Elmer spectrometer with a universal attenuated total reflectance (UATR) accessory; the acquisition parameters were: $8000-4000 \mathrm{~cm}^{-1}$, $4 \mathrm{~cm}^{-1}$ resolution, 1 gain and 40 accumulations. The relation between the epoxy group intensity band $\left(4523 \mathrm{~cm}^{-1}\right)$ and reference band $\left(4623 \mathrm{~cm}^{-1}\right)$. The reference band selected is the combination of the two stretching modes, $\mathrm{C}-\mathrm{H}$ and $\mathrm{C}=\mathrm{C}$, of the phenyl group of the epoxy group. They were analyzed in order to determine the water absorption by the samples. The water content $\left(w_{T}\right)$ was calculated by Equation 5:

$$
w_{t}=0,096+0,587 \frac{A_{w, t}-A_{w, 0}}{A_{r e f}}
$$

where $A_{W, 0}$ is the integrated absorbance related to the non-exposed sample to moisture, $A_{W T}$ is the integrated absorbance of sample exposed to moisture for time equals to $\mathrm{t}$ and $A_{R E F}$ is the integrated absorbance of the reference band. Gonzales-Benito and co-workers described this procedure in details as well the meaning of the constants of the Equation $5^{[24]}$.
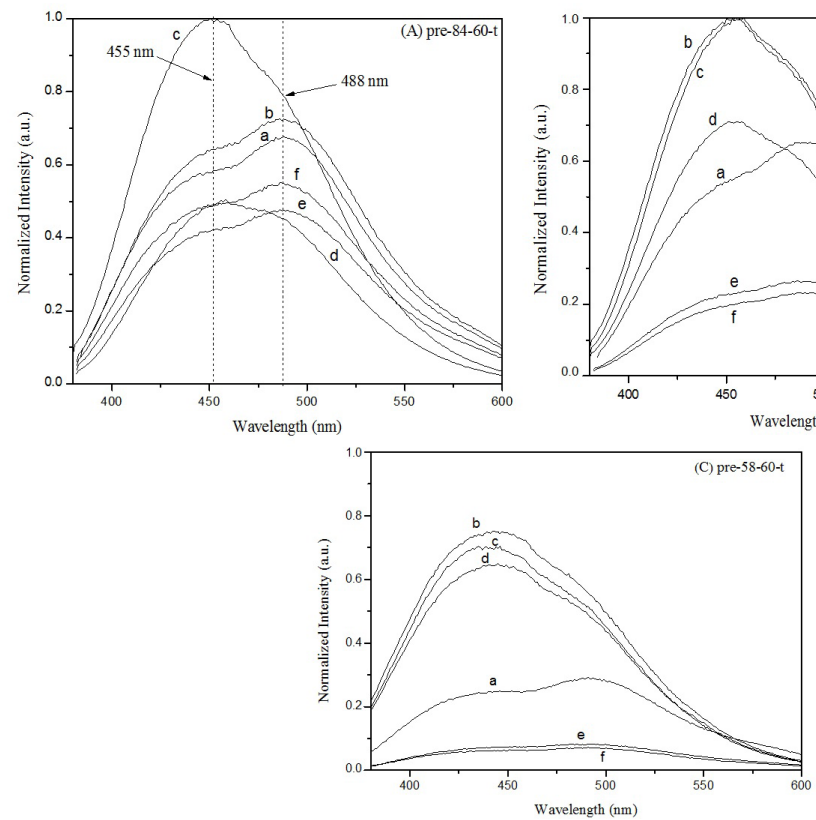

\subsection{Emission and excitation spectra}

Fluorescence spectra were measured with a steady-state luminescence spectrometer (FS920-Edinburgh Analytical Instruments) in the photocounting mode with a xenon arc lamp $450 \mathrm{~W}$ (Osram Co.) and double holographic grating monochromators of excitation and emission (Czerny- Turner configurations). The fluorescence spectra of the solid samples were obtained through a front-face illumination. The range of wavelength of excitation (290-380 nm) was chosen taking into account that the curing agent groups shows emission band after this region. The first moment of luminescence $(<v>)$, defined by the weighted average wavenumber (Equation 6) ${ }^{[25]}$, was used in order to analyze the water absorption into F-161 prepregs and laminates:

$$
\langle v\rangle=\frac{\sum_{i} v_{i} I\left(v_{\mathrm{i}}\right)}{\sum_{i} I\left(v_{\mathrm{i}}\right)}
$$

where $I\left(v_{P}\right)$ is the relative emission intensity at wavenumber $v_{I}$.

\section{Results and Discussions}

\subsection{F-161 prepreg samples under different humidities at 60 and $80^{\circ} \mathrm{C}$}

Figure 1A shows the spectra of electronic emission of the samples pre-84-80-t. These samples presented two superimposed emission bands: one centered at range $451-455 \mathrm{~nm}$ and another centered at $488 \mathrm{~nm}$. The emission bands in the region from $400-600 \mathrm{~nm}$ of pre-relative humidity-60-t samples can be attributed to the curing agent, which probably contains amine groups ${ }^{[26]}$. The band profile is very similar to the emission band of the cured prepreg sample. Sales et al. ${ }^{[2]}$ have discussed that the spectra of the

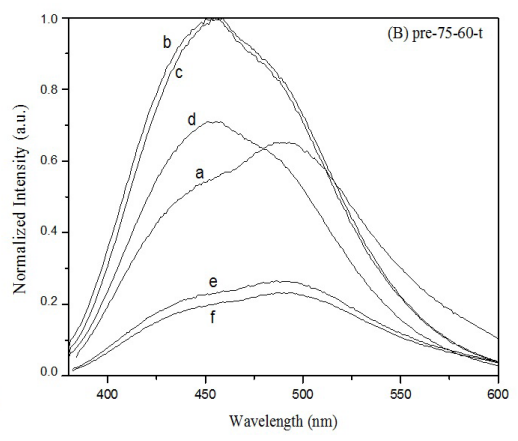

Figure 1. Electronic emission spectra of the samples $\left(\lambda_{\text {exc }}=370 \mathrm{~nm}\right)$ : (A) pre-84-60-t, (B) pre-75-60-t and (C) pre-58-60-t in the following periods of time ( $\mathrm{t}$ in days): (a) 1, (b) 7, (c) 15, (d) 30, (e) 60 and (f) 90. 
samples non-totally cured showed a red shift of maximum emission due to the conversion of primary amines into secondary and tertiary amines. As might be expected, the sample pre-84-80-1 (Figure 1A.a) showed no significant influence of water absorption in the polymeric matrix. The band centered at $488 \mathrm{~nm}$ is the most intense one for samples with $\mathrm{t}=1,7,60$ and 90 days, while the band centered in the range $451-455 \mathrm{~nm}$ is the most intense for samples with $\mathrm{t}=15$ and 30 days. Another remarkable observation is that the displacements were dependent on the exposure time. Therefore, one can observe a displacement towards to the blue region for exposure time from 7 to 15 days and another displacement towards to the red region for periods of time of 30 to 60 days.

The emission spectrum of pre-84-60-7 (Figure 1A.b) does not present a significant change when compared to the pre-84-60-1. However, the spectrum of the sample pre-84-60-15 (Figure 1A.c) when compared to that sample pre-84-60-7 shows a considerable increase in the relative intensity of the maximum emission and a blue shift to $455 \mathrm{~nm}$. The relative intensity of the emission band of sample pre-84-60-30 (Figure 1A.d) is lower than samples pre-84-60-15 and its maximum is centered at $455 \mathrm{~nm}$. Sample pre-84-60-60 and pre-84-60-90 show emission bands whose profiles are very similar to the band of the pre-84-60-1, but there is a decreasing of the relative intensities. Emission spectra of samples pre-75-60-t (Figure 1B) and pre-58-60-t (Figure 1C) are very similar with spectra of the pre-84-60-t samples; the only difference is related to exposure time where the blue shift is observed. The emission spectrum of sample pre-84-60-t shows a shift towards the blue region with 15 and 30 days, while those related to samples pre-75-60-t and pre-58-60-t show the same shift after a period of time of 7,15 and 30 days.

These two effects on the photophysical behavior of samples subjected to moisture at intermediate times can be related. The relative emission intensity increases when there is an increase in the radiative rate constant of the fluorophores, which can be attributed to the stiffness of the polymeric matrix. On the other hand, the blue shift can be related to an important change of the polarity of the medium, which could influence the fluorophore photophysical behavior. According to Lakowicz ${ }^{[27]}$, a decrease in the medium polarity can lead to a blue shift of maximum emission. On other hand, the interaction with a more polar solvent could lead to the stabilization of the excited electronic state, shifting the emission maximum to the red region. Since the emission band was attributed to secondary and tertiary amine compounds, one can assume that water molecules diffuse through the polymeric matrix and form hydrogen bonds with the amine and hydroxyl groups. The effect of water diffusion through the polymeric matrix in the photophysical behavior of the fluorophores is the increase in energy of its excited electronic state and consequent shift of the maximum emission to the blue region. The shift to the blue region could indicate that the formation of hydrogen bonds of water with polar groups reduces its polarity. In this case, water would act as a pseudo curing agent and would contribute to an increase in the stiffness of the polymeric matrix, which is related to an increase in the rate constants of radiative processes and consequent increase in emission intensity, as observed in the spectra of samples.

Novolac epoxy matrix of F-161 prepreg type is a very polar material, since it contains a considerable number of hydroxyl ${ }^{[25]}$. These groups result from the opening of the epoxy rings by crosslinking reaction. The water molecule could act as a pseudo-curing agent by its interaction with hydroxyl groups of the different polymer chains. This interaction increases the polymer matrix stiffness and increases the radiative decay rate constant. Consequently, an increase in the relative intensity of the luminescence emission is observed for very short times of exposure to moisture. According to Soles et al. ${ }^{[28]}$, resin polarity is of primary importance in determining the equilibrium moisture uptake. Less polar resins, such as the non-amine series, absorb very little water compared to the amine-containing materials. Accompanying polarity, topology has a secondary effect on uptake. A greater intrinsic hole volume fraction $\left(V_{0}\right)$ leads to an increase in the equilibrium moisture content. $V_{0}$ is isothermally elevated by increasing crosslink density, the consequence of which is enhancement of the effective polarity. Steric hindrances at the crosslink junctions introduce unoccupied volume elements coincident with the polar hydroxyls and amines. $V_{0}$ is localized to regions immediately adjacent to crosslink junctions. Calculations indicate there will be approximately $7 \times 10^{20}$ nanopores per $\mathrm{cm}^{3}$ for epoxy matrix and $2 \times 10^{21}$ polar groups per $\mathrm{cm}^{3}$. If a typical equilibrium uptake (for the bifunctional resins) of $3 \mathrm{wt} \%$ is assumed, approximately $1 \times 10^{21}$ water molecules per $\mathrm{cm}^{3}$ of resin will be absorbed ${ }^{[28,29]}$.

The decrease in the relative intensity of samples treated for longer periods of time (30 and 60 days) denotes an increasing of the rate constant of non-radiative decay that can be related to the plasticization effects of the polymer matrix due to water uptake. This process is associated to the amount of unbound water that occupies the free volume of the polymeric matrix. On the other hand, higher water content in the bulk increases the medium polarity, resulting in the red shift of the emission band. Sung and Sung et al. ${ }^{[19]}$ verified that water absorption induces changes in the medium polarity and a decrease in the relative intensity of the emission band luminescence. According to Zhou and Lucas ${ }^{[30]}$, there are two ways that water molecules can bind to the epoxy matrix. In the first one, the absorbed water can diffuse into the polymer chain breaking the van der Waals bonds, resulting in an increase of the chain segment mobility and swelling. Water molecule interacts strongly with hydrophilic functional groups such as hydroxyl or amine in epoxy resin. The second one occurs when the absorbed water molecule connects to the polymer chain forming pseudo or secondary crosslinking. This process turns the secondary amine groups into tertiary amines, increasing the conversion degree.

The results of this work strongly suggest that water first acts as a pseudo curing agent, since it was observed an increasing of the emission intensity. Then occurs plasticization of the polymeric matrix related to decreasing of the emission intensity. It must be clear that this photophysical behavior was observed for all samples submitted to different relative humidities treated at $60{ }^{\circ} \mathrm{C}$. 
Prepreg samples submitted to relative humidities of 84 (Figure 2A), 75 (Figure 2B) and 58\% (Figure 2C) at $80{ }^{\circ} \mathrm{C}$ showed emission bands with the same profiles and a wavelength maximum at $488 \mathrm{~nm}$, a band overlapped around $450 \mathrm{~nm}$ and there are no shift of the maximum emission bands. Comparing these spectra with those obtained for samples submitted to the same relative humidity at $60{ }^{\circ} \mathrm{C}$, it is observed that the bands have the same emission band profile than those submitted for longer times, in which the water content is higher.

First moment of fluorescence (Equation 6) allows the comparison of the emission spectra of the samples exposed to three conditions of relative humidity for different periods. This equation has been used for many authors not only to study the influence of water in polymeric matrix ${ }^{[24]}$, but also to study polymerization reaction ${ }^{[21,31]}$ and investigate the structure of elastomeric network ${ }^{[32]}$. Figure 3A shows the first moment $(<v>)$ versus exposure time for samples pre-84-60-t, pre-75-60-t and pre-58-60-t. For a better evaluation of water effects in epoxy matrix, all scales are in the same range. The sample pre-58-60-t showed the highest value for the luminescence band first moment $(\langle v\rangle)$ and the lowest one for water content. The variation of first moment of samples pre-58-60-1 and pre-58-60-7 has a significant difference of $857 \mathrm{~cm}^{-1}$, indicating that the absorbed water has an influence in polymeric matrix.

On the other hand, the values of water content and luminescence band first moment $(\langle v\rangle)$ for the samples pre-84-60-t and pre-75-60-t are very close (Figure 3A). The effect of increased emission intensity is much more pronounced in samples pre-58-60-7, pre-58-60-15 and pre-58-60-30 compared with the other samples (Figure 3A). However, the results of gravimetric analysis showed water contents between 0.1 and $0.3 \% \mathrm{~m} / \mathrm{m}$, which are half the water content of the samples exposed to relative humidity of $84 \%$ for the same periods of time (Figure 3B). The photophysical behavior of the samples pre-58-60-t can indicate that the influence of bound water is significant only for lower water contents. For higher water contents, one can conclude that the $\langle v\rangle$ variation is inversely proportional to the water content.

Figures $3 \mathrm{~A}$ and $3 \mathrm{~B}$ show the first moment of luminescence $(<v>)$ and water content of the samples pre-58-80-t, pre-75-80-t and pre-84-80-t, respectively. It can be observed that $\langle v\rangle$ does not change significantly for samples submitted at $80{ }^{\circ} \mathrm{C}$, in relation to the same parameter for samples treated at $60{ }^{\circ} \mathrm{C}$. According to Sales ${ }^{[33]}$, the increase in the relative luminescence emission intensities and in the rate constant of radiative decay is due to the increased rigidity of the polymer matrix. Probably, the water forms hydrogen bonds with polar groups of the polymer matrix, acting as pseudo-curing agent by exposure time periods for each specific humidity, but as the rate of diffusion of water in the polymer matrix should be greater than $80^{\circ} \mathrm{C}$ (Table 1), it is assumed that the matrix plasticization occurs in a shorter time period (between 7 to 30 days) when the samples are submitted to the higher relative humidity and temperature.

Table 1 summarizes the wavelengths of the emission bands, water contents, first moments of luminescence and diffusion coefficients of prepreg F161 samples at 60 and $80^{\circ} \mathrm{C}$. Sample pre-58-60 treated for 15 days presented the higher first moment of luminescence $-21986 \mathrm{~cm}^{-1}$. The emission maximum of this sample was shifted to the blue region at
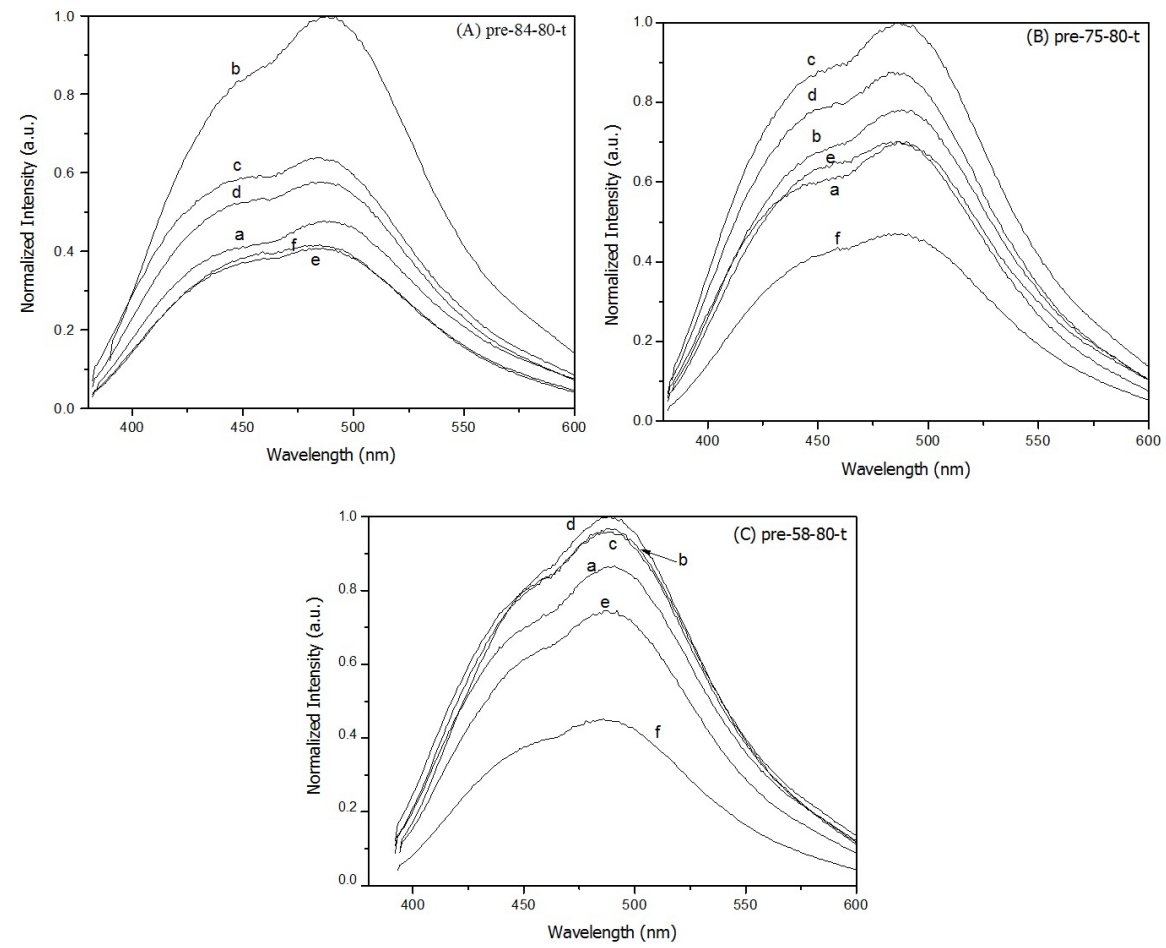

Figure 2. Electronic emission spectra of the samples $\left(\lambda_{\text {exc }}=370 \mathrm{~nm}\right.$ ): (A) pre-84-80-t, (B) pre-75-80-t and (C) pre-58-80-t in the following periods of time ( $\mathrm{t}$ in days): (a) 1, (b) 7, (c) 15, (d) 30, (e) 60 and (f) 90. 

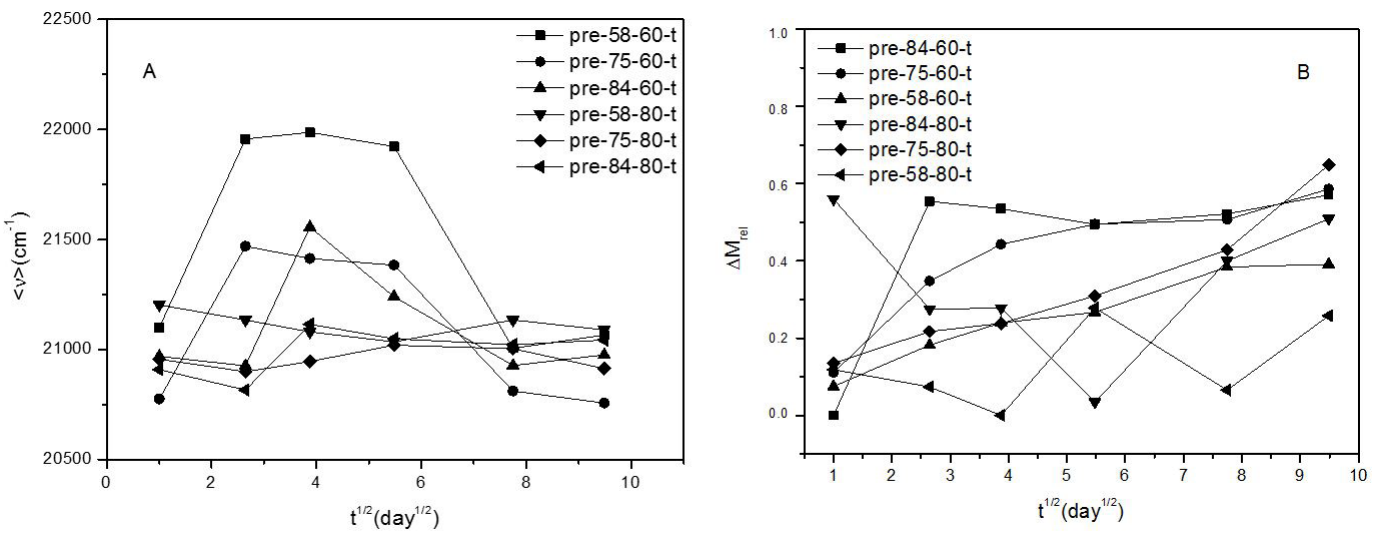

Figure 3. F-161 prepreg samples at 60 and $80^{\circ} \mathrm{C}$ : (A) First moment of luminescence $<\mathrm{v}>$ versus exposure time and (B) Dependence of the water content with exposure time.

Table 1. Summary of wavelengths of the emission bands, water content, first moment of luminescence and diffusion coefficients of prepreg F161 samples at 60 and $80^{\circ} \mathrm{C}$.

\begin{tabular}{|c|c|c|c|c|c|}
\hline Samples & & & $\begin{array}{c}\text { Water content } \\
\left(\Delta \mathbf{M}_{\text {rel }} \%\right)\end{array}$ & $\begin{array}{c}\text { First moment of } \\
\text { fluorescence }\left(\mathrm{cm}^{-1}\right)\end{array}$ & $\begin{array}{c}\text { Diffusion coefficient } \\
\left(\mathrm{cm}^{2} / \mathrm{min}\right)\end{array}$ \\
\hline \multirow{7}{*}{ pre- $84-60-\mathrm{t}^{\mathrm{a}}$} & 452 & 488 & & & \multirow{7}{*}{$1.22475 \times 10^{-7}$} \\
\hline & & 1 & 0.00 & 20969 & \\
\hline & & 7 & 0.55 & 20926 & \\
\hline & $15^{\mathrm{b}}$ & & 0.54 & 21556 & \\
\hline & 30 & & 0.49 & 21240 & \\
\hline & & 60 & 0.52 & 20927 & \\
\hline & & 90 & 0.57 & 20975 & \\
\hline \multirow{6}{*}{ pre-75-60-t } & & 1 & 0.08 & 20775 & \multirow{6}{*}{$2.01633 \times 10^{-8}$} \\
\hline & $7^{\mathrm{b}}$ & & 0.35 & 21469 & \\
\hline & 15 & & 0.44 & 21413 & \\
\hline & 30 & & 0.49 & 21383 & \\
\hline & & 60 & 0.50 & 20811 & \\
\hline & & 90 & 0.59 & 20757 & \\
\hline \multirow{6}{*}{ pre-58-60-t } & & 1 & 0.08 & 21100 & \multirow{6}{*}{$2.0742 \times 10^{-8}$} \\
\hline & $7^{\mathrm{b}}$ & & 0.18 & 21957 & \\
\hline & 15 & & 0.24 & 21986 & \\
\hline & 30 & & 0.27 & 21922 & \\
\hline & & 60 & 0.38 & 21005 & \\
\hline & & 90 & 0.39 & 21066 & \\
\hline \multirow{6}{*}{ pre- $84-80-\mathrm{t}^{\mathrm{a}}$} & & 1 & 0.56 & 20909 & \multirow{6}{*}{$5.1126 \times 10^{-8}$} \\
\hline & & $7^{\mathrm{b}}$ & 0.28 & 20816 & \\
\hline & & 15 & 0.28 & 21115 & \\
\hline & & 30 & 0.03 & 21049 & \\
\hline & & 60 & 0.40 & 21023 & \\
\hline & & 90 & 0.51 & 21043 & \\
\hline \multirow{6}{*}{ pre-75-80-t } & & 1 & 0.14 & 20957 & \multirow{6}{*}{$1.61816 \times 10^{-9}$} \\
\hline & & 7 & 0.22 & 20900 & \\
\hline & & $15^{\mathrm{b}}$ & 0.24 & 20946 & \\
\hline & & 30 & 0.31 & 21020 & \\
\hline & & 60 & 0.43 & 21003 & \\
\hline & & 90 & 0.65 & 20913 & \\
\hline \multirow{6}{*}{ pre-58-80-t } & & 1 & 0.12 & 21203 & \multirow{6}{*}{$1.8963 \times 10^{-8}$} \\
\hline & & 7 & 0.07 & 21134 & \\
\hline & & 15 & 0 & 21081 & \\
\hline & & $30^{\mathrm{b}}$ & 0.28 & 21035 & \\
\hline & & 60 & 0.07 & 21134 & \\
\hline & & 90 & 0.26 & 21089 & \\
\hline
\end{tabular}


$452 \mathrm{~nm}$ and was the more intense one. The water content and diffusion coefficient were $0.24 \%$ and $2.0742 \times 10^{-8} \mathrm{~cm}^{2} / \mathrm{min}$, respectively. Samples treated at 75 and $84 \%$ of relative moisture at $60{ }^{\circ} \mathrm{C}$ for 15 days presented maximum first moments of luminescence of 21413 and $21556 \mathrm{~cm}^{-1}$ both lower than that of the sample pre-58-60-15. The water contents for samples pre-75-60-15 and pre-84-60-15 were of 0.44 and $0.54 \%$. The water content increases as the increase of the relative humidity, as it was expected, but decreases the effect of water uptake in the first moment of luminescence. Samples pre-58-60-t and pre-75-60-t presented diffusion coefficients very close, but the first sample had the effect of increasing the emission intensity more pronounced. Results indicate that the sample contains a significant amount of bound water, since there is an increased relative intensity of the emission band related to the increased rigidity of the polymer matrix and shift of the emission band to the blue region, due to hydrogen bonding of water with amine and hydroxyl groups.

All the samples treated at $80^{\circ} \mathrm{C}$ present emission maxima at $488 \mathrm{~nm}$ and values of first moment of luminescence very close. Emission spectra of the samples pre-84-80-7 and pre-84-80-15 show an increase of the relative intensity of the band that could indicate an increase of the stiffness of

the polymeric matrix, but there is no noticeable change in the polarity of the medium, since it was not a blue shift. Values of diffusion coefficient were to close too. It should be emphasized that the gravimetric measurement of the sample pre-58-80-15 is unreliable since there is no mass change. It must be assumed that the samples treated at $80^{\circ} \mathrm{C}$ do not contain detectable amounts of bound water. Soles et al. ${ }^{[29]}$ have discussed that this behavior is partially understood in terms of the interactions between water and a polar group. Increasing the temperature increases the external heat and shifts the above reaction toward the formation of free water. The analysis of the luminescence spectra of the samples treated at $80^{\circ} \mathrm{C}$ confirms the conclusions of those authors. There is a great amount of free water in the samples that maintains a higher polarity of polymeric matrix.

\subsection{F-161 laminated samples under different humidities at $60^{\circ} \mathrm{C}$ e $80^{\circ} \mathrm{C}$}

Figure $4 \mathrm{~A}$ shows the intrinsic luminescence spectra of samples lam-84-60-t. The spectra related to samples lam-75-60-t (Figure 4C) and lam-58-60-t (Figure 4E) are quite similar to those of pre-84-60-t (Figure 1A). All spectra
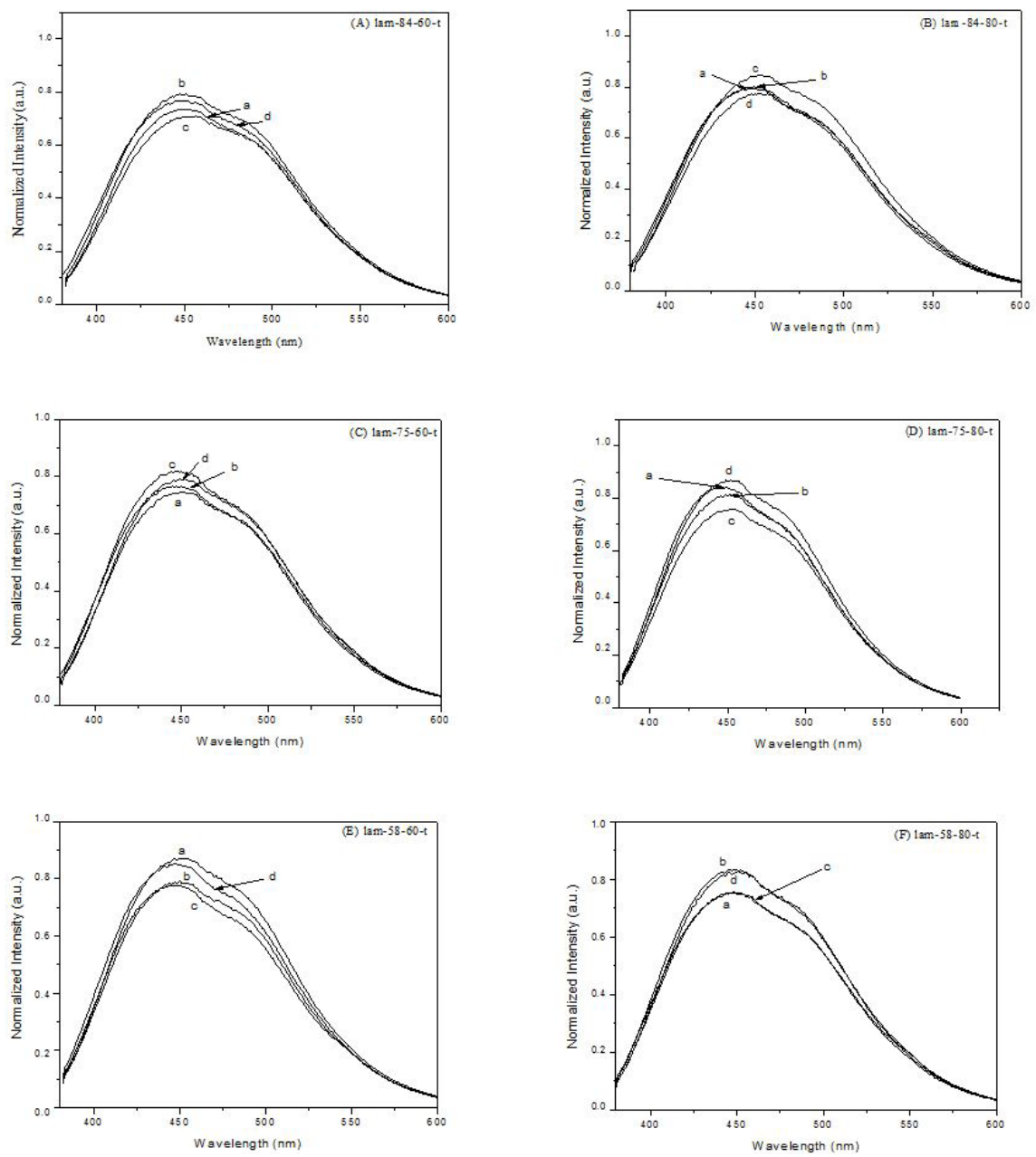

Figure 4. Electronic emission spectra of the samples (A) lam-84-60-t, (B) lam-84-80-t, (C) lam-75-60-t, (D) lam-75-80-t, (E) lam-58-60-t and (F) lam-58-80-t in the following periods of time (in days): (a) 1, (b) 7, (c) 15 and (d) 30. 
show the emission bands with the same profile and the wavelength of the maximum emission around $450 \mathrm{~nm}$, as well as the samples pre-84-60-t treated for 1, 7, 60 and 90 days. These bands are attributed to the emission of secondary and tertiary amine groups of the curing agent, which are covalently bound to the epoxy matrix. The emission band at $450 \mathrm{~nm}$ can indicate a reducing of the medium polarity related to hydrogen bonding of water with polar groups. All intrinsic luminescence spectra related to samples lam-84-80-t, lam-75-80-t and lam-58-80-t are quite similar and show emission bands with the same profile and the maximum emission of the sample lam-84-60-t do not showing significant changes.

Figures $5 \mathrm{~A}$ and $5 \mathrm{~B}$ show the luminescence first moment and the water content variations with the exposure time of the samples lam-84-60-t, lam-75-60-t, lam-58-60-t, lam-58-80-t, lam-75-80-t and lam-84-80-t, respectively. The dependence the values of first moment luminescence $(<v>)$ of the three samples on the root square of the exposure time is very small. The biggest difference between the higher value and the lower one was equal to $142 \mathrm{~cm}^{-1}$, while the curve related to the prepreg samples showed the major difference equal to $982 \mathrm{~cm}^{-1}$. This behavior was expected, since the amount of the free volume decreased during the curing and lamination process. This increase in the laminate bulk density decreases the capacity of water absorption. However, the matrix polarity does not change during the experiments, but the emission maximum is located at $448 \mathrm{~nm}$ and is attributed to bound water. This same photophysical behavior was observed mainly for the samples pre-58-60-7, pre-58-60-15, pre-58-60-30 and pre-75-60-7 and indicated the presence of bound water.

Table 2 summarizes wavelengths of the emission bands, water content, first moment of luminescence and diffusion coefficients of laminated F161 samples at 60 and $80{ }^{\circ} \mathrm{C}$. All spectra showed a maximum emission at $448 \mathrm{~nm}$ that indicate a less polar medium due to hydrogen bonding of water to amine and hydroxyl groups. However, samples of laminates not showed a significant increase in the relative emission intensity as observed for samples of prepreg. It can be inferred that the amount of bound water in the laminate is not enough to increase the rigidity of the polymer matrix. Water contents confirm this conclusion since they were much smaller compared with the samples of prepreg, as it was expected since laminated samples have a lower free volume. The values of the first moment of fluorescence showed no significant variations too. The values of the diffusion coefficients ranged from $2.021 \times 10^{-5}$ to $4.28 \times 10^{-5} \mathrm{~cm}^{2} / \mathrm{min}$ for samples lam-84-60, lam-75-60 and lam-58-60. Samples treated at $80{ }^{\circ} \mathrm{C}$ had diffusion coefficients varying from $4.9537 \times 10^{-7}$ to $1.233 \times 10^{-5} \mathrm{~cm}^{2} / \mathrm{min}$.

\subsection{FT-NIR spectroscopy}

Figure 6 shows FT-NIR spectrum of samples btt-pre, pre-84-60-90 and pre-84-80-90. Table 3 shows the main absorption bands, including epoxy, methylene, and phenyl groups. These assignments agreed with those from literature ${ }^{[34]}$ related to epoxy resin. Peaks at $6064 \mathrm{~cm}^{-1}$ and at $4524 \mathrm{~cm}^{-1}$ are related to the epoxy ring ${ }^{[35]}$ and they can be attributed to ${ }^{[35,36]}$ : i) the first overtone of the terminal $\mathrm{CH}_{2}$ stretching mode; ii) to a combination band of the second overtone of the epoxy ring stretching at $916 \mathrm{~cm}^{-1}$ with the fundamental $\mathrm{C}-\mathrm{H}$ stretching at about $2725 \mathrm{~cm}^{-1}$. Table 3 shows the attribution of the vibrational modes of the FT-NIR bands of the prepreg F-161. The band at $4524 \mathrm{~cm}^{-1}$ can be attributed to a combination of the stretching fundamental $\left(3050 \mathrm{~cm}^{-1}\right)$ with the $\mathrm{CH}_{2}$ deformation fundamental $\left(1460 \mathrm{~cm}^{-1}\right)$ of the epoxy ring ${ }^{[37-39]}$.

The band at $5238 \mathrm{~cm}^{-1}$ is related to water absorption process by epoxy resin ${ }^{[40]}$ and can also be attributed to a combination band of groups $-\mathrm{CH}$ and $-\mathrm{CH}_{2}{ }^{[41]}$. The intensity of the band at $5238 \mathrm{~cm}^{-1}$ of the sample exposed to moisture at 60 and $80{ }^{\circ} \mathrm{C}$ (Figure 6B) did not show an increase of intensity when compared with the btt-pre band; this band did not make the water absorption analysis possible. The Novolac epoxy resin structure shows an oxirane group attached to each benzene ring, consequently the density of $\mathrm{OH}$ group of this resin is higher than other epoxy resins. Therefore, the band at $4524 \mathrm{~cm}^{-1}$ is the best for monitoring the water uptake in the polymeric matrix, since it is related to the epoxy ring. According Gonzalez-Benito et al. ${ }^{[2]}$, when water molecule enters into the epoxy resin, some molecules may interact with the functional groups of resin, since the resin may not be fully cured ${ }^{[24]}$. Water molecule is considered a curing agent of the "Lewis base" type ${ }^{[42]}$. The anion generated by the reaction between the amine and
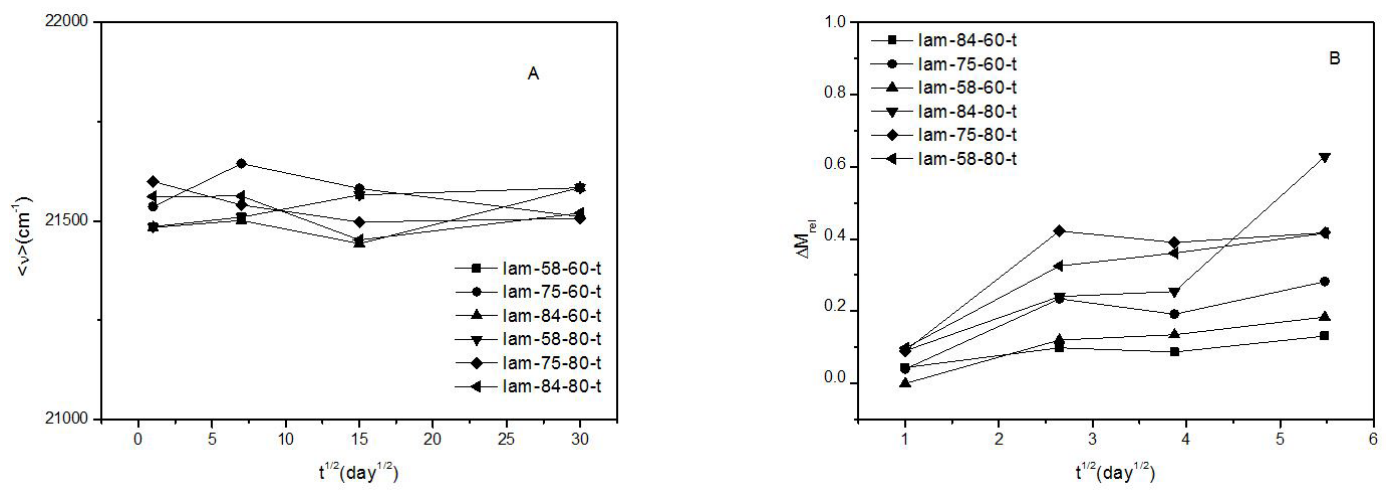

Figure 5. F-161 laminated samples at 60 and $80^{\circ} \mathrm{C}$ : (A) First moment of luminescence $<\mathrm{v}>$ versus exposure time and (B) Dependence on the water content with exposure time. 
Table 2. Summary of wavelengths of the emission bands, water content, first moment of luminescence and diffusion coefficients of laminated F161 samples at 60 and $80^{\circ} \mathrm{C}$.

\begin{tabular}{|c|c|c|c|c|}
\hline Samples & $\begin{array}{l}\lambda_{\max } \\
(\mathrm{nm})\end{array}$ & $\begin{array}{c}\text { Water content } \\
\left(\Delta \mathrm{M}_{\text {rel }} \%\right)\end{array}$ & $\begin{array}{c}\text { First moment of } \\
\text { fluorescence }\left(\mathrm{cm}^{-1}\right)\end{array}$ & $\begin{array}{c}\text { Diffusion coefficient } \\
\left(\mathrm{cm}^{2} / \mathrm{min}\right)\end{array}$ \\
\hline & 448 & 478 & & \\
\hline \multirow{4}{*}{ lam-84-60- $\mathrm{t}^{\mathrm{a}}$} & 1 & 0.044 & 21483 & \multirow{4}{*}{$3.3803 .10^{-5}$} \\
\hline & $7^{\mathrm{b}}$ & 0.098 & 21502 & \\
\hline & 15 & 0.087 & 21443 & \\
\hline & 30 & 0.131 & 21584 & \\
\hline \multirow{4}{*}{ lam-75-60-t } & 1 & 0.040 & 21536 & \multirow{4}{*}{$2.0220 .10^{-5}$} \\
\hline & 7 & 0.234 & 21644 & \\
\hline & $15^{\mathrm{b}}$ & 0.192 & 21582 & \\
\hline & 30 & 0.282 & 21510 & \\
\hline \multirow{4}{*}{ lam-58-60-t } & $1^{\mathrm{b}}$ & 0.000 & 21486 & \multirow{4}{*}{$4.2864 .10^{-5}$} \\
\hline & 7 & 0.120 & 21510 & \\
\hline & 15 & 0.135 & 21566 & \\
\hline & 30 & 0.184 & 21584 & \\
\hline \multirow{4}{*}{ lam-84-80- $\mathrm{t}^{\mathrm{a}}$} & 1 & 0.091 & 21562 & \multirow{4}{*}{$4.9537 .10^{-7}$} \\
\hline & 7 & 0.241 & 21563 & \\
\hline & $15^{\mathrm{b}}$ & 0.255 & 21452 & \\
\hline & 30 & 0.628 & 21520 & \\
\hline \multirow{4}{*}{ lam-75-80-t } & 1 & 0.091 & 21599 & \multirow{4}{*}{$1.2333 .10^{-5}$} \\
\hline & 7 & 0.422 & 21540 & \\
\hline & 15 & 0.390 & 21498 & \\
\hline & $30^{\mathrm{b}}$ & 0.417 & 21506 & \\
\hline \multirow{4}{*}{ lam-58-80-t } & 1 & 0.098 & 21486 & \multirow{4}{*}{$5.8829 .10^{-6}$} \\
\hline & $7^{\mathrm{b}}$ & 0.325 & 21510 & \\
\hline & 15 & 0.361 & 21566 & \\
\hline & 30 & 0.416 & 21584 & \\
\hline
\end{tabular}

${ }^{\mathrm{a}} \mathrm{t}=$ exposure time; ${ }^{\mathrm{b}}$ most intense band.

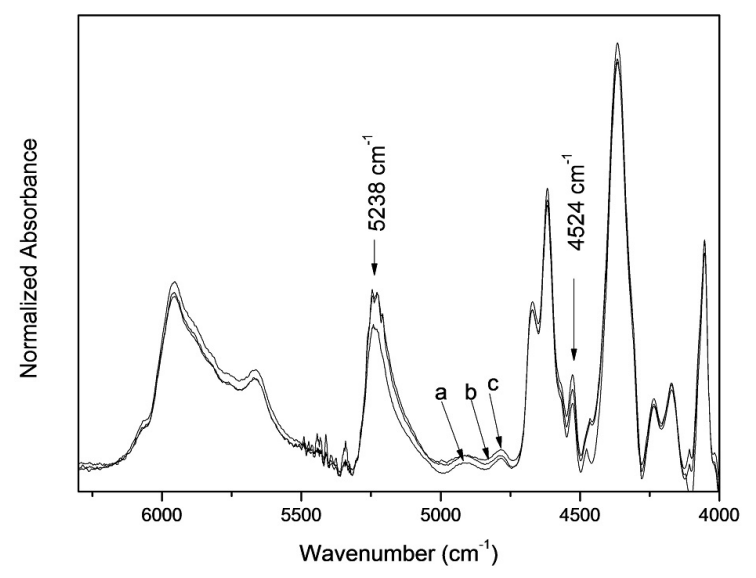

Figure 6. FT-NIR spectra of samples: (a) btt-pre (b) pre-84-60-90 and (c) pre-84-80-90.

the epoxy ring can react with an active hydrogen present in water, alcohol, phenol or carboxyl to form a new anion. This anion is able to open a second epoxy ring continuing the curing reaction ${ }^{[10,42-46]}$.

Figure 7A shows the absorbed water content by the samples of F-161 prepreg samples submitted to moisture of 84,75 and $58 \%$ at $60{ }^{\circ} \mathrm{C}$. There is a significant increase in the water content during periods of time ${ }^{1 / 2}$ of 2.6, 3.9 and 5.5. In the following periods of time ${ }^{1 / 2}$ there is an abrupt decrease in intensity, because of the stabilization of the quantity of absorbed water by samples. This behavior suggests that in the early periods, the water binds to epoxide groups increasing the intensity of the band at $4524 \mathrm{~cm}^{-1}$ and, in the subsequent period of time, water molecule acts as a curing agent, promoting the opening of the epoxide ring supporting the curing process. Comparing the results obtained by the weight measurements and water content measured by FT-NIR, one can observe the same behavior: an increase in the earlier periods of time due to water absorption and, in the subsequent periods a stabilization of water content due to the crosslink process promoted by water. 
Table 3. Attribution of the normal mode vibrations of the NIR absorption spectra of prepreg samples.

\begin{tabular}{cl}
\hline Wavenumber $\left(\mathbf{c m}^{-1}\right)$ & \multicolumn{1}{c}{ Band assignments } \\
\hline 6071 & First overtone of terminal (methylene)-CH fundamental stretching vibration (epoxy ring). \\
\hline 5961 & Overtone of the $\mathrm{CH}$ axial deformation of the phenyl group. \\
\hline 5886 & First overtones of the fundamental $-\mathrm{CH}_{2}$ and $-\mathrm{CH}$ stretching vibration. \\
\hline 5661 & $-\mathrm{OH}$ combination band. \\
\hline 5247 & $-\mathrm{CH},-\mathrm{CH}_{2}$ combination band. \\
\hline 4672 & $\begin{array}{l}\text { Combination band of the aromatic conjugated } \mathrm{C}=\mathrm{C} \text { stretching }\left(=1625 \mathrm{~cm}^{-1}\right) \text { with the aromatic }-\mathrm{CH} \text { fundamental } \\
\text { stretching }\left(=3050 \mathrm{~cm}^{-1}\right) .\end{array}$ \\
\hline 4625 & $\begin{array}{l}\text { Combination band of the aromatic conjugated } \mathrm{C}=\mathrm{C} \text { stretching }\left(=1625 \mathrm{~cm}^{-1}\right) \text { with the aromatic }-\mathrm{CH} \text { fundamental } \\
\text { stretching }\left(=3050 \mathrm{~cm}^{-1}\right) .\end{array}$ \\
\hline 4524 & $\begin{array}{l}\text { Conjugated epoxy } \mathrm{CH} \text { deformation band }\left(=1460 \mathrm{~cm}^{-1}\right) \text { with the aromatic }-\mathrm{CH} \text { fundamental stretch }\left(=3050 \mathrm{~cm} \mathrm{~cm}^{-1}\right), \\
-\mathrm{NH} \text { absorption band. }\end{array}$ \\
\hline 4480 & $-\mathrm{CH}_{2}-\mathrm{CH}_{2}$ combination band. \\
\hline 4362 & $-\mathrm{CH}_{2}$ combination band. \\
\hline 4216 & $-\mathrm{CH}_{2}-\mathrm{CH}_{2}$ combination band. \\
\hline 4155 & Aromatic $-\mathrm{CH}$ combination band. \\
\hline 4058 & Aromatic combination band. \\
\hline
\end{tabular}
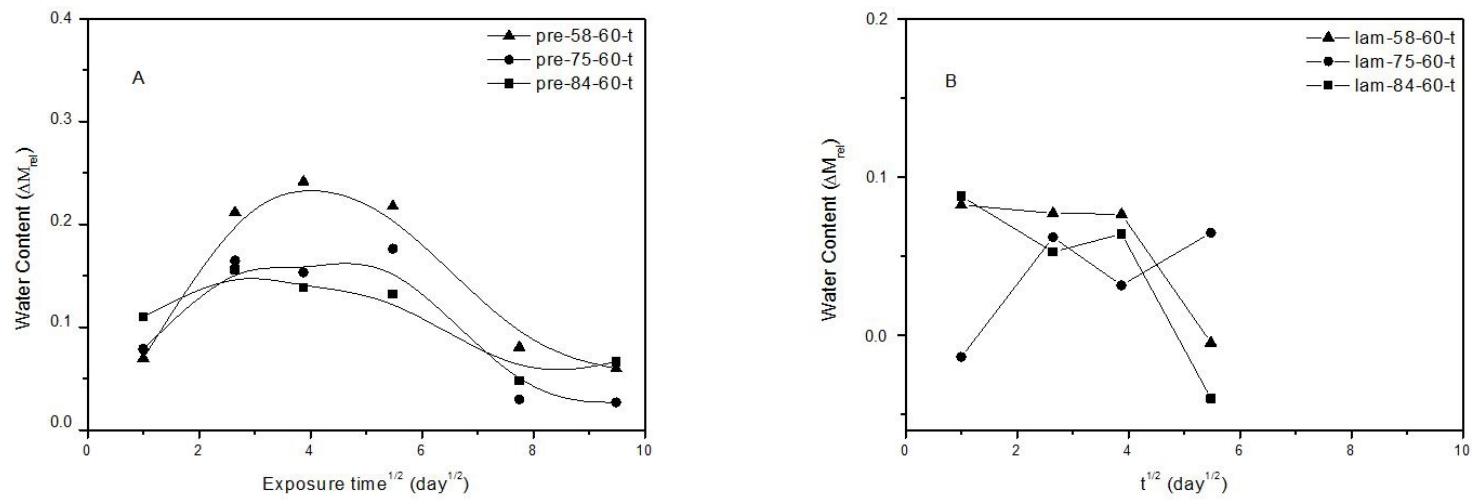

Figure 7. Water content versus time measured by FT-NIR: (A) F-161 prepreg samples at $60^{\circ} \mathrm{C}$ for the following periods of time (in days ${ }^{1 / 2}$ ): 1.0, 2.6, 3.9, 5.5, 7.7 and 9.5 and (B) F-161 laminates samples at $60^{\circ} \mathrm{C}$, for the following periods of time (in days ${ }^{1 / 2}$ ): 1.0, 2.6, 3.9 and 5.5.

Figure 7B shows the amount of water absorbed by F-161 laminates samples submitted to relative humidity of 84,75 and $58 \%$ at $60{ }^{\circ} \mathrm{C}$ measured by FT-NIR, respectively. It can be claimed that no significant changes in the water content of the laminates (Figure 5). This behavior was expected because laminate samples were already cured and this process decreases free volume of polymeric matrix. Therefore, there is a greater compression of the polymer chains and less capacity to absorb water.

\section{Conclusions}

All samples of prepreg F-161, independent on relative humidity and exposure time, showed very similar photophysical behavior: (a) a blue shift of the maximum emission and (b) an increase in relative intensity of luminescence. This photophysical behavior can be attributed to a change of the medium polarity.

The decrease in the relative intensity of luminescence in the spectra of samples treated for longer times (30,60 and 90 days) may be related to the plasticization process of the polymer matrix performed by water molecules. The results of this work strongly suggest that water first acts as an pseudo curing agent, since it was observed an increasing of the emission intensity and then occurs plasticization of the polymeric matrix related to decreasing of the emission intensity. It must be clear that this photophysical behavior was observed for all samples submitted to different relative humidities. This photophysical behavior had not been previously observed by other authors

The first moment of luminescence $(v)$ variation is inversely proportional to the quantity of water absorbed by the polymer matrix. Prepreg samples results indicated that the polarity of prepreg samples change during the experiments, characterized by the red shift of the emission spectra. On the other hand, it is not observed either a red shift emission or an increasing of water content in laminated samples, due to the curing process conditions of temperature and pressure.

The results provided by FT-NIR of samples treated for short periods of time showed an increase in the intensity of the band $4524 \mathrm{~cm}^{-1}$. This increase is related to hydrogen bonding between the absorbed water molecules and epoxy groups and confirms the presence of bound water observed through luminescence spectroscopy. 


\section{Acknowledgements}

The authors thank Instituto de Aeronáutica e Espaço (IAE), Fundação de Apoio à Pesquisa do Estado de São Paulo (FAPESP) and Coordenação de Aperfeiçoamento de Nível Superior (CAPES) (Brazil).

\section{References}

1. Clark, G., Saunders, D. S., van Blaricum, T. J., \& Richmond, M. (1990). Moisture absorption in graphite/ epoxy laminates. Composites Science and Technology, 39(4), 355-375. http:// dx.doi.org/10.1016/0266-3538(90)90081-F.

2. Apicella, A., Tessieri, R., \& Cataldis, C. (1984). Sorption modes of water in glassy epoxies. Journal of Membrane Science, 18 , 211-225. http://dx.doi.org/10.1016/S0376-7388(00)85035-8.

3. Ellis, T. S., \& Karasz, F. E. (1984). Interactions of epoxy resins with water: the depression of glass transition temperature. Polymer, 25(5), 664-669. http://dx.doi.org/10.1016/00323861(84)90034-X.

4. Jelinski, L. W., Dumais, J. J., Cholli, A. L., Ellis, T. S., \& Karasz, F. E. (1985). Nature of the water-epoxy interaction. Macromolecules, 18(6), 1091-1095. http://dx.doi.org/10.1021/ ma00148a008.

5. Woo, M., \& Piggot, M. (1987). Water absorption of resins and composites: I. epoxy homopolymers and copolymers. Journal Composites Technology Research, 9(3), 101-107. http://dx.doi. org/10.1520/CTR10249J.

6. Adamson, M. J. (1980). Thermal expansion and swelling of cured epoxy resin used in graphite/epoxy composite materials. Journal of Materials Science, 15(7), 1736-1745. http://dx.doi. org/10.1007/BF00550593.

7. Li, L., Yu, Y., Su, H., Zhan, G., Li, S., \& Wu, P. (2010). The diffusion mechanism of water transport in amine-cured epoxy networks. Applied Spectroscopy, 64(4), 458-465. PMid:20412632. http://dx.doi.org/10.1366/000370210791114220.

8. Cotugno, S., Larobina, D., Mensitieri, G., Musto, P., \& Ragosta, G. (2001). A novel spectroscopic approach to investigate transport processes in polymers: the case of water-epoxy system. Polymer, 42(15), 6431-6438. http://dx.doi.org/10.1016/ S0032-3861(01)00096-9.

9. Roy, S., \& Xu, W. (2001). Modeling of diffusion in the presence of damage in polymer matrix composites. International Journal of Solids and Structures, 38(1), 115-126. http://dx.doi. org/10.1016/S0020-7683(00)00006-8.

10. Choi, H. S., Ahn, K. J., Nam, J.-D., \& Chun, H. J. (2001). Hygroscopic aspects of epoxy/carbon fiber composite laminates in aircraft environments. Composites. Part A, Applied Science and Manufacturing, 32(5), 709-720. http://dx.doi.org/10.1016/ S1359-835X(00)00145-7.

11. Patel, S. R., \& Case, S. W. (2000). Durability of a graphite/ epoxy woven composite under combined hygrothermal conditions. International Journal of Fatigue, 22(9), 809-820. http://dx.doi.org/10.1016/S0142-1123(00)00041-4.

12. Zafar, A., Bertocco, F., Schjødt-Thomsen, J., \& Rauhe, J. C. (2012). Investigation of the long term effects of moisture on carbon fibre and epoxy matrix composites. Composites Science and Technology, 72(6), 656-666. http://dx.doi.org/10.1016/j. compscitech.2012.01.010.

13. Asp, L. E. (1998). The effects of moisture and temperature on the interlaminar delamination toughness of a carbon/epoxy composite. Composites Science and Technology, 58(6), 67-977. http://dx.doi.org/10.1016/S0266-3538(97)00222-4.

14. Chateauminois, A., Vincent, L., Chabert, B., \& Soulier, J. P. (1994). Study of the interfacial degradation of a glass epoxy composite during hygrothermal aging using water diffusion measurements and dynamicmechanical thermal-analysis. Polymer, 35(22), 4766-4779. http://dx.doi.org/10.1016/00323861(94)90730-7.

15. Majerus, M. S., Soong, D. S., \& Prausnitz, J. M. (1984). Experimental measurements and monte-carlo simulation of water diffusion into epoxy matrices. Journal of Applied Polymer Science, 29(8), 2453-2466. http://dx.doi.org/10.1002/ app.1984.070290803.

16. Li, Y., Miranda, J., \& Sue, H.-J. (2001). Hygrothermal diffusion behavior in bismaleimide resin. Polymer, 42(18), 7791-7799. http://dx.doi.org/10.1016/S0032-3861(01)00241-5.

17. Dang, W., \& Sung, N. H. (1994). In-situ cure monitoring of diamine cured epoxy by fiberoptic fluometry using extrinsic reactive fluorophore. Polymer Engineering and Science, 34(9), 707-715. http://dx.doi.org/10.1002/pen.760340903.

18. Sung, C. S. P., Pyun, E., \& Sun, H. L. (1986). Characterization of epoxy cure by UV-visible and fluorescence spectroscopy: azo chromophoric labeling approach. Macromolecules, 19(12), 2922-2934. http://dx.doi.org/10.1021/ma00166a008.

19. Sung, C. S. P., \& Sung, N. H. (1993). Fluorescence characterization of cure and water uptake in polymers and composites. Materials Science and Engineering A, 162(1-2), 241-247. http://dx.doi. org/10.1016/0921-5093(90)90049-9.

20. Pyun, E., \& Sung, C. S. P. (1991). Network structure in diaminecured tetrafunctional epoxy by UV-Visible and fluorescence spectroscopy. Macromolecules, 24(4), 855-861. http://dx.doi. org/10.1021/ma00004a007.

21. Paik, H. J., \& Sung, N. H. (1994). Fiberoptic intrinsic fluorescence for in-situ cure monitoring of amine cured epoxy and composites. Polymer Engineering and Science, 34(12), 1025-1032. http://dx.doi.org/10.1002/pen.760341212.

22. American Society for Testing and Materials - ASTM. (2012). ASTM D5229/D229M-12. West Conshohocken: ASTM.

23. Soles, C. L., Chang, F. T., Gidley, D. W., \& Yee, A. F. (2000). Contributions of the Nanovoid Structure to the Kinetics of Moisture Transport in Epoxy Resins. Journal of Polymer Science. Part B, Polymer Physics, 38(5), 776-791. http://dx.doi. org/10.1002/(SICI)1099-0488(20000301)38:5<776::AIDPOLB15>3.0.CO;2-A.

24. Olmos, D., López-Morón, R., \& González-Benito, J. (2006). The nature of the glass fibre surface and its effect in the water absorption of glass fibre/epoxy composites: the use of fluorescence to obtain information at the interface. Composites Science and Technology, 66(15), 2758-2768. http://dx.doi. org/10.1016/j.compscitech.2006.03.004.

25. Gonzalez-Benito, J., Bravo, J., Mikes, F., \& Baselga, J. (2003). Fluorescence labels to monitor water absorption in epoxy resins. Polymer, 44(3), 653-659. http://dx.doi.org/10.1016/ S0032-3861(02)00806-6.

26. Sales, R. C. M., Diniz, M. F., Dutra, R. C. L., Thim, G. P., \& Dibbern-Brunelli, D. (2010). Thermal Curing of Glass-Epoxy Prepregs by Luminescence Spectroscopy. Journal of Applied Polymer Science, 17(2), 664-671. http://dx.doi.org/10.1002/ app.31953.

27. Lakowicz, J. R. (1999). Principles of fluorescence spectroscopy (2nd ed.). New York: Kluwer Academic.

28. Soles, C. L., \& Yee, A. F. (2000). Discussion of the Molecular Mechanisms of Moisture Transport in Epoxy Resins. Journal of Polymer Science. Part B, Polymer Physics, 38(5), 792-802. http:// dx.doi.org/10.1002/(SICI)1099-0488(20000301)38:5<792::AIDPOLB16>3.0.CO;2-H.

29. Soles, C. L., Chang, F. T., Bolan, B. A., Hristov, H. A., Gidley, D. W., \& Yee, A. F. (1998). Contributions of the nanovoid structure to the moisture absorption properties of epoxy resins. Journal of Polymer Science. Part B, Polymer Physics, 
36(17), 3035-3048. http://dx.doi.org/10.1002/(SICI)10990488(199812)36:17<3035::AID-POLB4>3.0.CO;2-Y.

30. Zhou, J., \& Lucas, J. P. (1999). Hygrothermal effects of epoxy resin. Part I: the nature of water in epoxy. Polymer, 40(20), 5505-5512. http://dx.doi.org/10.1016/S0032-3861(98)00790-3.

31. Quirin, J. C., \& Torkelson, J. M. (2003). Self-referencing fluorescence sensor for monitoring conversion of nonisothermal polymerization and nanoscale mixing of resin components. Polymer, 44(2), 423-432. http://dx.doi.org/10.1016/S00323861(02)00780-2.

32. Leezenberg, P. B., \& Frank, C. W. (1995). Selective sorption and solvation in dansyl-labeled poly(dimethylsiloxane) networks swollen in binary solvent mixtures. Macromolecules, 28(22), 7407-7415. http://dx.doi.org/10.1021/ma00126a019.

33. Sales, R. C. M., \& Brunelli, D. D. (2005). Luminescence spectroscopy applied to a study of the curing process of diglycidyl-ether of bisphenol-A (DGEBA). Materials Research, 8(3), 299-304. http://dx.doi.org/10.1590/S151614392005000300013.

34. Poisson, N., Lachenal, G., \& Sautereau, H. (2000). Nearand mid-infrared spectroscopy studies of an epoxy reactive system. Vibrational Spectroscopy, 12, 565-574. http://dx.doi. org/10.1016/0924-2031(96)00027-6.

35. Musto, P., Mascia, L., Ragosta, G., Scarinzi, G., \& Villano, P. (2000). The transport of water in a tetrafunctional epoxy resin by near-infrared Fourier transform spectroscopy. Polymer, 41(2), 565-574. http://dx.doi.org/10.1016/S0032-3861(99)00210-4.

36. Chike, K. E., Myrick, M. L., Lyon, R. E., \& Angel, S. M. (1993). Raman and near-infrared studies of an epoxy resin. Applied Spectroscopy, 47(10), 1631-1635. http://dx.doi. org/10.1366/0003702934334714.

37. Lachenal, G., Pierre, A., \& Poisson, N. (1996). FT-NIR Spectroscopy: trends and application to the kinetic study of Epoxy/triamine system (Comparison with DSC and SEC Results). Micron, 27(5), 329-334. http://dx.doi.org/10.1016/ S0968-4328(96)00022-4.

38. Wang, Q., Storm, B. K., \& Houmoller, L. P. (2003). Study of the isothermal curing of an epoxy prepregs by near-infrared spectroscopy. Journal of Polymer Science, 87, 2295-2305. http://dx.doi.org/10.1002/app.11711.

39. Rigail-Cedeño, A., \& Sung, C. S. P. (2005). Fluorescence and IR characterization of epoxy cured with aliphatic amines. Polymer, 46(22), 9378-9384. http://dx.doi.org/10.1016/j. polymer.2005.04.063.

40. González-Benito, J. (2003). The nature of the structural gradient in epoxy curing at a glass/fiber epoxy matrix interface using FT-IR imaging. Journal of Colloid and Interface Science, 267(2), 326-332. PMid:14583208. http://dx.doi.org/10.1016/ S0021-9797(03)00550-2.

41. Hepburn, D. M., Kemp, I. J., \& Cooper, J. M. (2000). Degradation of filled epoxy resin surfaces. Polymer Degradation and Stabilization, 70(2), 245-251. http://dx.doi.org/10.1016/ S0141-3910(00)00120-8.

42. Lee, H., \& Neville, K. (1967). Handbook of epoxy resin. New York: McGraw-Hill.

43. Shaw, S. E., Russo, T., Solomon, D. H., \& Qiao, G. G. (2006). An alternative pathway for the hydrolysis of epoxy ester compounds. Polymer, 47(25), 8247-8252. http://dx.doi. org/10.1016/j.polymer.2006.10.004.

44. Li, L., Zhang, S. Y., Chen, Y. H., Liu, M. J., Ding, Y. F., Luo, X. W., Pu, Z., Zhou, W. F., \& Li, S. (2005). Water transportation in epoxy resin. Chemistry of Materials, 17(4), 839-845. http:// dx.doi.org/10.1021/cm048884z.

45. Jana, R. N., \& Bhunia, H. (2008). Higrothermal degradation of the composites laminates from woven carbon SC-15 epoxy resin and woven glass/SC-15 epoxy resin. Polymer Composites, 29(6), 664-669. http://dx.doi.org/10.1002/pc.20417.

46. Zhao, C., \& Whalen, D. L. (2006). Transition state effects in the acid-catalyzed hydrolysis of 5-methoxyacenaphthylene 1,2-oxide: implications for the mechanism of acid-catalyzed hydrolysis of cyclopenta[cd]pyrene 3,4-oxide. Chemical Research in Toxicology, 19(2), 217-222. PMid:16485897. http://dx.doi.org/10.1021/tx050281u.

Received: May 23, 2016

Revised: Aug. 19, 2016

Accepted: Dec. 20, 2016 